\title{
A Synopsis of Current Malaria Diagnosis Trends
}

\author{
James Chipeta ${ }^{1,2}$, Sungano Mharakurwa ${ }^{3}$, Philip Thuma ${ }^{3}$ and Nirbhay Kumar ${ }^{4}$ \\ 1. University of Zambia School of Medicine, Department of Paediatrics and Child Health, P.0. Box 50110, \\ Lusaka, Zambia \\ 2. The School of Medicine and University Teaching Hospital Malaria Research Unit (SMUTH-MRU), \\ University Teaching Hospital, Department of Paediatrics and Child Health, D-Block, P/B RW1X, \\ Lusaka, Zambia \\ 3. Malaria Institute at Macha (MIAM), P.O. Box 630166, Choma, Zambia \\ 4. Molecular Microbiology and Immunology, Bloomberg School of Public Health, Johns Hopkins \\ University, Baltimore, MD 21205, USA
}

\begin{abstract}
Malaria has remained a major cause of morbidity and mortality in the under developed and developing countries of the tropical and subtropical regions of the world. Globally 3.3 billion people live in areas where malaria exists, affecting 300-500 million people annually and it is estimated to be killing approximately 1-3 million people each year and $90 \%$ of these mortalities occur in African children especially in sub Saharan Africa. Currently, although several control methods are beginning to result in downward trends in incidence in some countries, the gross number of malaria cases is still on the increase due to several factors including poor and ineffective diagnosis. Prompt and effective diagnosis is essential for the management and control of malaria. Over the years evidence has shown that traditional methods for diagnosing malaria remain problematic with a number of limitations. In this synoptic review an update of malaria diagnosis is presented and discussed highlighting the limitations and difficulties of both clinical (symptoms/ clinical signs-based) and laboratory (parasite-based) diagnosis of malaria. Enhancement of accurate malaria diagnosis is now
\end{abstract}

\section{Corresponding Author:}

James Chipeta

Senior Lecturer and Honorary Consultant

University of Zambia, School of Medicine

Department of Paediatrics and Child Health

P.O. Box 50110, Lusaka, ZAMBIA

Telephone: 260-211-254965 or Cell: 260-955-834198

Fax:260-211-250305

E-mail: Jameschipeta@smuth-mru.org.zm or damaseke@yahoo.com more imperative than ever not only in the background of the current new era of malaria treatment with relatively expensive artemisininbased combination therapies (ACTs), but more so in the heightened global campaign to effectively control, manage and possibly eradicate malaria from the face of the globe.

\section{INTRODUCTION}

The onslaught of the human race by malaria over the centuries is well documented in history and it appears the scourge has been menacing mankind from time immemorial. ${ }^{1-3}$ The oldest fossil puts the age of Plasmodium species at 2-10 million years and evolution of the most virulent malaria parasite, Plasmodium falciparum, is estimated to be about 100 000 years ago. This is at least more than 100 times older than the Broken Hill man. ${ }^{1-4}$ The oldest classical and systematic clinical description of the disease was by the ancient father of medicine, Hippocrates, who between 460-377 B.C accurately gave a description of the quartian nature of the malaria fever. ${ }^{5}$ Various holy scriptures including the Hindu scriptures ${ }^{6-7}$ also give detailed description of malaria episodes in ancient times suggesting that, indeed, this scourge has been menacing mankind from time immemorial. Interestingly, while similar ancient scourges, such as small pox and plagues have been eradicated or at least controlled to insignificant levels, malaria continues to affect human civilization by hundreds of millions in number of people being affected. It is endemic in the developing world with 3.3 billion of the world's population being at risk of the disease 
every year. It is estimated that annually 300-500 million people suffer from malaria and 1-3 million deaths, world wide, are attributable to malaria and $90 \%$ of these deaths occur in African children especially in sub Saharan Africa. ${ }^{8}$ In Zambia malaria has recently been estimated to be causing 4 million clinical episodes and about 8000 deaths annually. ${ }^{9}$

Indeed, although various control methods are beginning to result in downward trends in incidences in some countries ${ }^{10}$, the gross number of malaria cases is still on the increase due to several factors including poor and ineffective diagnosis. ${ }^{11}$ Cumulative research evidence over the past years has indicated that effective diagnosis reduces both morbidity and mortality from malaria. ${ }^{12-22}$ Hence, currently, there is heightened need to reinforce practical malaria diagnostics world-wide for effective control and management of malaria. In this synopsis an update of malaria diagnosis is presented and discussed highlighting the limitations and difficulties of both clinical (symptoms and clinical signs-based) and laboratory (parasitebased) diagnosis of malaria. Enhancement of accurate malaria diagnosis is now more imperative than ever not only in the background of the current new era of malaria treatment with relative expensive ACTs, but more so in the heightened global campaign to effectively control, manage and possibly eradicate malaria from the face of the globe.

\section{CLINICAL DIAGNOSIS}

Clinical diagnosis of malaria is based on patient's signs and symptoms from history and physical evaluation. It is the least expensive of all methods of malaria diagnosis and is widely practiced. ${ }^{13}, 24$ However, clinical diagnosis of malaria is at all times quite challenging and has various limitations due to indistinguishable nature of the disease from other illnesses with similar signs and symptoms, such as pneumonia, sepsis, the human immunodeficiency virus (HIV) infection, influenza, etc. ${ }^{24-31}$ Indeed though clinical diagnosis has sensitivity as high as $100 \%$ its specificity is quite low ranging from 0 $9 \% .^{32-35}$ This non-specific nature of malaria symptoms and signs entails that in malaria-endemic areas, there is over-diagnosis leading to overtreatment of malaria and non-treatment of other illnesses (see Figure 1, for illustrative case).
Figure 1. A 13 year old Child Presumed to have malaria Black water fever.
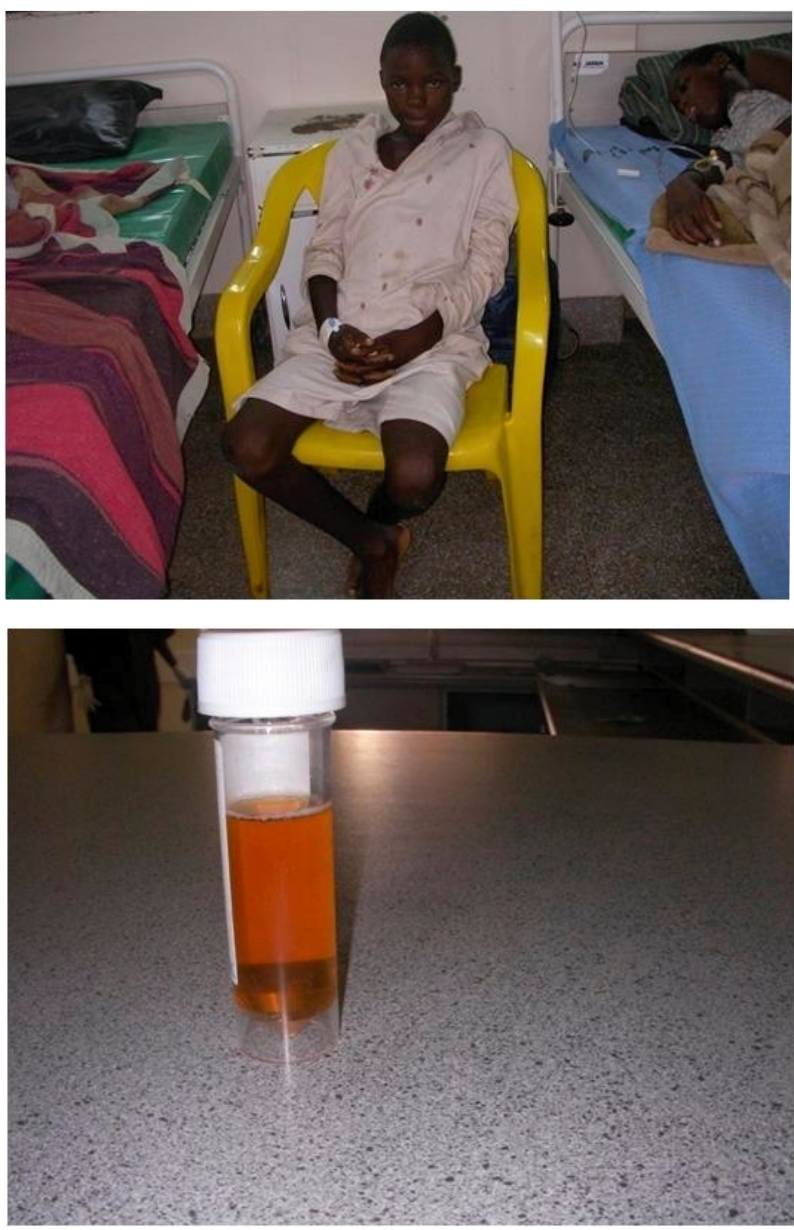

A 13 year old boy was referred to University Teaching Hospital (UTH) on $16^{\text {th }}$ July 2009 after an empirical treatment with Fansidar for malaria at a peripheral clinic that was presumed to have complicated into Blackwater fever (Note his haematuric urine sample). Subsequent investigations and clinical evaluation at UTH revealed the boy to be G6PD deficient with negative malaria parasite slide as well as a negative RDT.

Meanwhile, on the other hand, there is misdiagnosis in non-endemic areas. Thus, reliance on clinical diagnosis alone results in a number of problems as demonstrated by many reports ${ }^{36-42}$ including; i) over diagnosis of malaria, ii) unnecessary morbidity and mortality as other illnesses end up being under diagnosed and under treated, iii) over treatment and needless wastage of health resources being spent on non-malaria cases, iv) inappropriate prescription of anti-malarials contributing to anti-malarial drug resistance, and (v) neglecting treatment of other 
clinical conditions with similar symptoms. It is thus imperative that clinical diagnosis should not be solely relied on but be enhanced by combining it with laboratory based diagnosis.

\section{LABORATORY DIAGNOSIS}

Currently there is a range of different laboratory techniques to confirm and diagnose malaria including the traditional microscopy techniques, rapid malaria diagnostic tests (RDTs) and molecular techniques such as the polymerase chain reaction (PCR). Most of these are being certified by the World Health Organization (WHO) for wider routine usage. ${ }^{43}$ Overall, and as evidenced by research locally ${ }^{44}$ and elsewhere ${ }^{45-47}$ laboratory or parasite-based malaria diagnosis is more costeffective compared to clinical diagnosis in that it ensures appropriate drug use and, if prudently utilized, reduces malaria and other disease related morbidity. However, like clinical diagnosis, laboratory diagnostic techniques on their own have limitations with regard to; i) sensitivity, ii) specificity, iii) accuracy, iv) precision, v) time consumed, vi) cost-effectiveness, vii) labor intensiveness, viii) the need for skilled microscopists, and ix) the problem of inexperienced health workers (both technicians and clinicians).

\section{Microscopy}

Microscopy, to date, has remained the 'goldstandard' of malaria diagnosis since Laveran made the first microscopic demonstration and identification of the malaria causative agent, Plasmodium, in $1880 .{ }^{14}$ The technique has had very little change with minor improvements of staining techniques by Romanowsky in the $19^{\text {th }}$ Century. The conventional microscopy diagnosis is by staining (Giemsa, Wright's or Field's stains) thin and thick peripheral blood smears. The technique is widely accepted worldwide due to its simplicity, low cost, high specificity and ability to assess parasite density. Studies continue to demonstrate its benefit in aiding effective management of malaria in endemic countries such as Sub-Saharan Africa particularly in this region of Southern Africa. ${ }^{45}$ Though microscopy has high specificity; its sensitivity is unfortunately very low especially at low parasite density. A study in Tanzania among 10 hospitals showed that over a third $(39 \%)$ of the so diagnosed positive slides were actually false positive. ${ }^{48}$ Another similar study in Kenya of 17 outpatient clinics showed that the positive predictive value of 'positive' slides was only $22 \%$. Indeed, O'Meara et al, have also demonstrated that even among highly trained microscopy personnel there is high inter-expert discrepancy in malaria microscopy parasite readings. ${ }^{47}$ To counter-balance these limitations, continued retraining of personnel, such as microscopists, is necessary and in some instances such efforts have demonstrated positive results. ${ }^{48}$ In addition, traditional routine microscopy can be reinforced by other concentration techniques such as the Quantitative Buffy Coat (QBC). However, overall, microscopy still poses a lot of challenges including labor intensiveness of the technique, low sensitivity, need of high diagnostic expertise, and requirement of minimum infrastructure (microscope, power/electricity, etc).

\section{Rapid Diagnostic Tests (RDTs)}

The World Health Organization (WHO) has recently endorsed and ushered in RDTs as the new simple, quick, accurate and cost effective diagnostic tests for determining the presence of malaria parasites and thus providing a suitable alternative to microscopy in the effective and prompt diagnosis of malaria. ${ }^{43}$ RDTs currently available in the market are quite a few ${ }^{49}$ and include brands such as OptiMAL, Paracheck, ICT, para-sight-F, parascreen, and SD Bioline. Unlike the various microscopy techniques, RDTs do not require laboratory equipment and are all based on the same principle and detect malaria antigen in blood flowing along a membrane containing specific antimalaria antibodies (see Figure 2). Most of the available RDTs are $P$. falciparum protein specific (either histidine rich protein II -HRP-II or lactase dehydrogenase-LDH) while some RDTs detect $P$. falciparum and other Plasmodium proteins such as aldolase or pan-malaria pLDH. Several studies have reported the performance of RDTs to be excellent. ${ }^{33-}$ ${ }^{34,50-52}$ Inarguably, RDTs are enhancing the benefits of parasite-based diagnosis of malaria though not without problems or limitations. RDT limitations currently reported and experienced ${ }^{14,49}$ include variation in sensitivity, inability to be used as 'stand alone' diagnostic test, lack of community and Health worker confidence in them as recently documented locally ${ }^{53}$ and elsewhere ${ }^{54}$ and failure to detect mixed malaria infections. 
Figure 2.Plasmodium falciparum Histidine Rich Protein II (HRP-II) Based Rapid Malaria Diagnostic Test (RDT)

\section{A. Various parts of an RDT Test Strip}

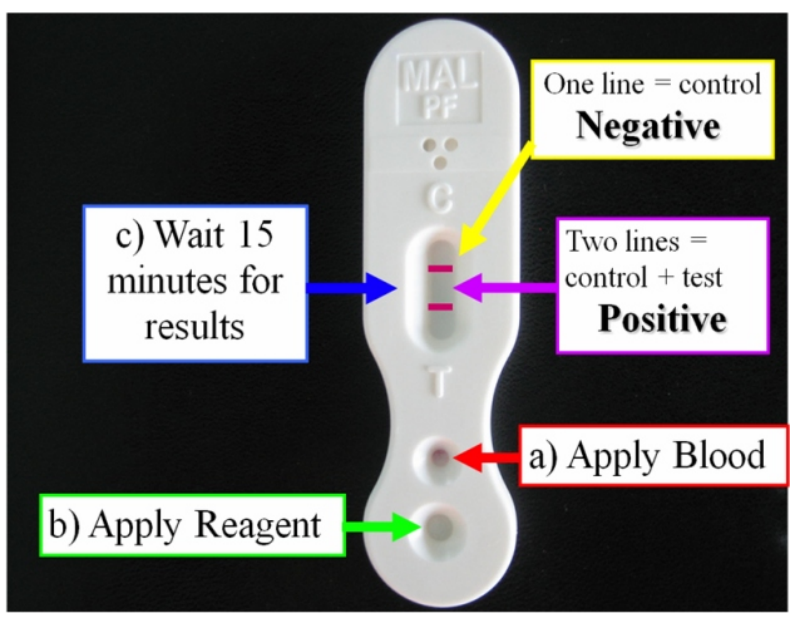

B.An Actual RDT test Result: Positive and Negative test.

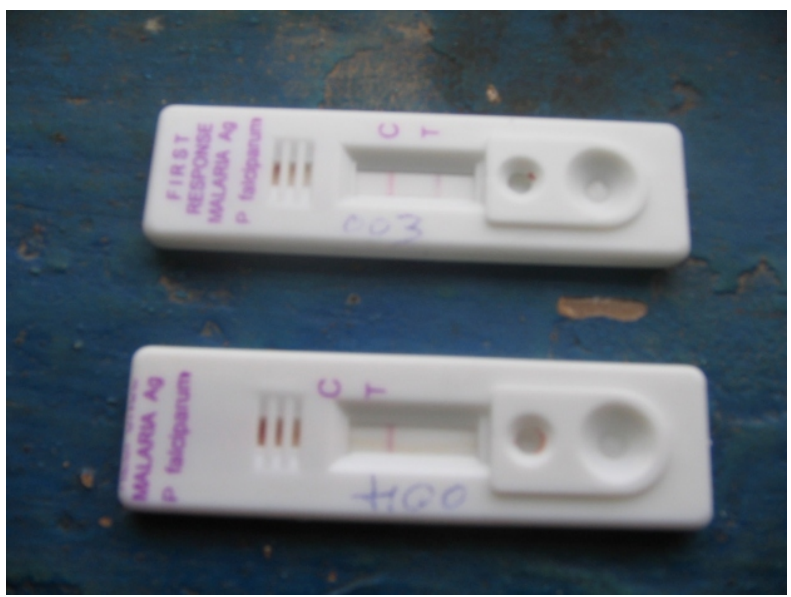

\section{Malaria Serological tests}

Serology malaria diagnostic tests utilize the principle of detecting antibodies against asexual blood stage malaria parasites. Among these tests, immunofluorescence antibody testing (IFA) is reportedly the most reliable one. ${ }^{55}$ IFA titres of $>1: 20$ are said to be positive, $<1: 20$ is said to be unconfirmed malaria and $>1: 200$ titres are classified as recent infections. ${ }^{56}$ IFA is simple, sensitive but time consuming and requires an expensive fluorescent microscope together with trained personnel.

\section{Molecular Malaria laboratory diagnostic tests}

Molecular malaria techniques such as PCR on blood or, more recently, even on saliva samples devised in Zambia by Mharakurwa et $\mathrm{al}^{57}$, the loop-mediated isothermal amplification (LAMP), microarray, mass spectrometry (MS), and flow cytometry (FCM) assay techniques are all new developments mainly utilized in research settings than during routine patient care. ${ }^{13}$ These malaria molecular techniques are, however, offering novel ways and strategies in malaria diagnosis.

\section{CONCLUDING REMARKS: RATIONAL APPROACHTO MALARIA DIAGNOSIS}

Current evidence indicates that no single method for the diagnosis of malaria is perfect nor can any one of them be a stand-alone accurate and effective diagnostic criterion (Table 1). Accurate and effective malaria diagnosis should thus involve a rational approach to each patient with suspected malaria employing both symptoms/signs-based and laboratory-based malaria diagnostic methods. The prioritizing of any of the malaria diagnostic methods, at all times, should be influenced by various factors including malaria endemicity, transmission pattern, the urgency of the diagnosis, the experience of the health worker, effectiveness of the health care system, and available budget resources.

\section{A C K N O W L E D G M E N T S A N D DECLARATION}

The authors have no conflict of interest to declare in this article but only to acknowledge that this review paper is based on the lecture given by the lead author at an NIH Fogarty International centre grant (2D43TW001587-06A2) sponsored 2009 Malaria Training and Research Capacity Building in Southern Africa (MTCBSA) international workshop held at MIAM, Macha, Zambia. 
Table1. Summary of Comparative analysis of Sensitivity and Specificity of Various Malaria Diagnosis

\begin{tabular}{llll}
\hline \multicolumn{1}{c}{ Malaria Diagnosis method } & Sensitivity & Specificity & References \\
\hline Clinical (Symptoms \& clinical signs-based) & $0-100 \%$ & $0-9 \%$ & $13,32,33,35$ \\
& & & \\
Microscopy & $0-40 \%$ & $66-91 \%$ & $13,42,48,47,58$ \\
& & & \\
Malaria Serological Tests & $68.2-95.5 \%$ & $92.2-96.1 \%$ & $13,55,56$ \\
& & & \\
& $3-100 \%$ & $52-99.5 \%$ & $13,33,34,50,51,52$ \\
\hline
\end{tabular}

Note: The cited comparative analysis of sensitivity and specificity of the various diagnostic methods is across the various malaria parasite species

\section{REFERENCES}

1. Carter R and Mendis KN. Evolutionary and Historical Aspects of the Burden of Malaria. Clinical Microbiology Reviews 2002;15(4):564-594

2. Jones WHS.1909. Malaria and the Greek history. Manchester University Press, Manchester, United Kingdom.

3. Celli A. 1933. The history of Malaria in the Roman Campagna from ancient times. John Bales, Danielsson, London, United Kingdom.

4. Escalante AA, Freeland DE, Collins WE and Lal AA. The evolution of primate malaria parasites based on the gene encoding cytochrome $b$ from the linear mitochondrial genome. Pro. Natl Acad. Sci. USA 1998; 95:8124-8129

5. Hippocrates. (English translation by W.H.S Jones). 1923. Aris, Waters and places VII, p.83-85;XXIV, p. 133-137;Epidemics I, p. 181-211; Epidemics III p.251-257 vol IV, Aphorisms II, p. 115; Aphorisms III, p. 125153. Heinemann, London, United Kingdom; Putnam, New York, N.Y.

6. Bruce-Chwatt LJ. 1965. Paleogenesis and paleoepidemiology of primate malaria. Bull. W.H.O. 32: 363-387
7. Sigerist HE. 1951. A History of Medicine, Vol. 1. Primitive and archaic medicine. Oxford University Press. New York, N.Y.

8. WHO, World Malaria Report, 2008

9. Ministry of Health, Zambia, HIMS Bulletin 2006

10. Breman JG, Alilio MS, and Mills A. Conquering the intolerable burden of malaria: What's new, what's needed: A summary. Am J. Trop. Med. Hyg 2004; 71(suppl 2):1-15.

11. Kiszewski A, Teklehaimanot A. A review of the clinical and epidemiological burdens of epidemic malaria. Am J Trop Med Hyg 2004; 179suppl 2):128-135.

12. Bell DR, Jorgensen P, Christophel EM, Palmer KL. Malaria risk: estimation of the malaria burden. Nature 2005; 437: E3-E4

13. Tangpukdee T., Duangdee C., Wilairatana P., and Krudsood S., Malaria Diagnosis: A Brief Review. Korean J Parasitol 2009; 47(2): 93102

14. Bell D., Wongsrichanalai C, and Barnwell J. Ensuring quality and access for malaria diagnosis: how can it be achieved? Nature Rev. Microbiol 2006

15. Greenwood, B. M. et al. Mortality and morbidity from malaria among children in a rural area of The Gambia, West Africa. Trans. $R$. Soc. Trop. Med. Hyg 1987; 81:478-486. 
16. Berkley, J. A. et al. Use of clinical syndromes to target antibiotic prescribing in seriously ill children in malaria endemic area: observational study. BMJ 2005; 330:995

17. Carcillo, J. A. Reducing the global burden of sepsis in infants and children: a clinical practice research agenda. Pediatr. Crit. Care Med 2005; 6: S157-S164

18. Peters, R. P. et al. A prospective study of bloodstream infections as cause of fever in Malawi: clinical predictors and implications for management. Trop. Med. Int. Health 2004; 9: 928-934 (2004).

19. WHO. World Malaria Report 2005 (WHO and UNICEF, Geneva, 2005).

20. Hay, S. I., Guerra, C.A., Tatem, A. J., Atkinson, P. M. \& Snow, R. W. Urbanization, malaria transmission and disease burden in Africa. Nature Rev. Microbiol2005; 3: 81-90

21. WHO. Strategic Orientation Paper on Prevention and Control of Malaria (WHO, Geneva, 2005).

22. Looareesuwan S. Malaria. In: Looareesuwan S, Wilairatana P, Eds, Clinical Tropical Medicine. 1st ed. Bangkok, Thailand. Medical Media. 1999, p 5-10.

23. English M, Punt J, Mwangi I, McHugh K, Marsh K. Clinical overlap between malaria and severe pneumonia in Africa children in hospital. Trans R Soc Trop Med Hyg 1996; 90: 658-662.

24. Molyneux E, Walsh A, Phiri A, Molyneux M. Acute bacterial meningitis in children admitted to the Queen Elizabeth Central Hospital, Blantyre, Malawi in 1996-97. Trop Med Int Health 1998; 3: 610-618.

25. Akpede GO, Sykes RM. Malaria with bacteraemia in acutely febrile preschool children without localizing signs: coincidence or association/complication? J Trop Med Hyg 1993; 96: 146-150.

26. Berkley J, Mwarumba S, Bramham K, Lowe B, Marsh K, Bacteraemia complicating severe malaria in children. Trans $R$ Soc Trop Med Hyg 1999; 93: 283-286.

27. Berkley JA, Maitland K, Mwangi I, Ngetsa C, Mwarumba S, Lowe BS, Newton CR, Marsh K, Scott JA, English M. Use of clinical syndromes to target antibiotic prescribing in seriously ill children in malaria endemic area: observational study. BMJ 2005; 330: 995.

28. Evans JA, Adusei A, Timmann C, May J, Mack D, Agbenyega T, Horstmann RD, Frimpong E. High mortality of infant bacteraemia clinically indistinguishable from severe malaria. QJM 2004; 97: 591-597.
29. Kallander K, Nsungwa-Sabiiti J, Peterson S. Symptom overlap for malaria and pneumonia-policy implications for home management strategies. Acta Trop 2004; 90: 211-214.

30. English M, Berkley J, Mwangi I, Mohammed S, Ahmed M, Osier F, Muturi N, Ogutu B, Marsh $\mathrm{K}$, Newton CRHypothetical performance of syndrome-based management of acute paediatric admissions of children aged more than 60 days in a Kenyan district hospital. Bull World Health Organ 2003; 81: 166-173.

31. Jakka SR, Veena S, Atmakuri RM, Eisenhut M. Characteristic abnormalities in cerebrospinal fluid biochemistry in children with cerebral malaria compared to viral encephalitis. Cerebrospinal Fluid Res 2006; 3: 8.

32. Perkins BA, Zucker JR, Otieno J. Jafari HS, Paxton L, Redd SC, Nahlen BL, Schwartz B, Oloo AJ, Olargo C, Gove S, Campbell CC. Evaluation of an algorithm for integrated management of childhood illness in an area of Kenya with high malaria transmission. Bull World Health Organ 1997; 75: 33-42.

33. Weber MW, Mulholland EK, Jaffar S, Troedsson H, Gove S, Greenwood BM. Evaluation of an algorithm for the integrated management of childhood illness in an area with seasonal malaria in the Gambia. Bull World Health Organ 1997; 75: 25-32.

34. Tarimo DS, Minjas JN, Bygbjerg IC. Malaria diagnosis and treatment under the strategy of the integrated management of children illness (IMCI): relevance of laboratory support from the rapid immunochromatographic tests of ICT malaria P.f/P.v and OptiMAL.Ann Trop Med Parasitol 2001; 95: 437-444.

35. Kyabayinze DJ, Tibenderana JK, Odong GW, Rwakimari JB, Counihan H. Operational accuracy and comparative persistent antigenicity of HRP2 rapid diagnostic tests for Plasmodium falciparum malaria in a hyperendemic region of Uganda. Malar J2008; 7: 221

36. Reyburn H, Mbatia R, Drakeley C, Carneiro I, Mwakasungula E,Mwerinde O, Saganda K, Shao J, Kitua A, Olomi R, Greenwood BM, Whitty CJ. Overdiagnosis of malaria in patientswith severe febrile illness in Tanzania: a prospective study. BMJ 2004; 329: 1212.

37. Fenn B, Morris SS, Black RE. Comorbidity in childhood in northern Ghana: magnitude, associated factors, and impact on mortality. Int J Epidemiol 2005; 34:368-375.

38. Kumar S. Overdiagnosis of cerebral malaria in patients admitted with neurological dysfunction. QJM2003; 96: 688. 
39. Makani J, Matuja W, Liyombo E, Snow RW, Marsh K, Warrell DA. Admission diagnosis of cerebral malaria in adults in an endemic area of Tanzania: implications and clinical description. QJM2003; 96:355-362.

40. Oster N, Krause E, Hatz C.Towards a rational malaria management at district hospital level: exploratory case series of febrile adult patients in a holoendemic area of Tanzania. Trop Doct 2000; 30: 203-207.

41. Petti CA, Polage CR, Quinn TC, Ronald AR, Sande MA. Laboratory medicine in Africa: a barrier to effective health care. Clin Infect Dis 2006; 42: 377-382.

42. Zurovac D, Midia B, Ochola SA, English M, Snow RW. Microscopy and outpatient malaria case management among older children and adults in Kenya. Trop Med Int Health 2006; 11:432-440.

43. World Health Organization. Guidelines for the treatment of Malaria. 1st ed. Geneva, Switzerland. WHO. 2006, p 133-143.

44. Chanda P, Castillo-Riquelme M, and Masiye F. Cost-effectiveness analysis of the available strategies for diagnosing malaria in outpatient clinics in Zambia. Cost Effectiveness and Resource Allocation 2009,7:5 doi:10.1186/1478-7547-7-5

45. Ngasala B, Mubi M, Warsame M, Petzold MG, Massele AY, Gustafsson LL, Tomson G, Premji $\mathrm{Z}$, Bjorkman A. Impact of training in clinical and microscopy diagnosis of childhood malaria on antimalarial drug prescription and health outcome at primary health care level in Tanzania: a randomized controlled trial. Malar J2008; 7: 199.

46. Reyburn H, Mwangi R, Mwakasungula E, Chonya S, Mtei F, 2006. Assessment of paediatric care in district and Regional hospitals in Tanga and Kilimanjaro Regions, North East $\mathrm{T}$ a $\mathrm{nz}$ a n i a A a i 1 a b 1 e a $\mathrm{t}$ : http://www.1shtm.ac.uk/malaria/ MC website/Assessment paediatric inpatient care in Tanzania.pdf

47. O 'Meara WP., Remich S., Ogutu B., et al. Systematic Comparison Of Two Methods To Measure Parasite Density From Malaria Blood Smears. Parasitol Res 2006; 99: 500-504

48. Ohrt C., Obare p., Nanakorn A., et al. Establishing a malaria diagnostics centre of excellence in Kisumu, Kenya. Malaria Journal 2007, 6:79 doi:10.1186/1475-2875-6-79
49. World Health Organization. List of known commercially available antigen-detecting malaria RDTs. [Accessed November 12, 2008]. Available at: http://www.wpro.who.int/sites/rdt

50. Endeshaw T, Gebre T, Ngondi J, Graves PM, Shargie EB, Ejigsemahu Y, Ayele B, Yohannes G, Teferi T, Messele A, Zerihun M, Genet A, Mosher AW, Emerson PM, Richards FO. Evaluation of light microscopy and rapid diagnostic test for the detection of malaria under operational field conditions: a household survey in Ethiopia. Malar J 2008; 7: 118.

51. Murray CK, Bell D, Gasser RA, Wongsrichanalai C. Rapid diagnostic testing for malaria. Trop Med Int Health 2003; 8: 876-883.

52. Murray CK, Gasser RA Jr, Magill AJ, Miller RS. Update on rapid diagnostic testing for malaria. Clin Microbiol Rev 2008; 21:97-110.

53. Hamer DH, Ndhlovu N, Zurovac D, et al. Improved Diagnostic Testing and Malaria Treatment Practices in Zambia. JAMA 2007;297(20):2227-2231

54. Reyburn H, Mbakilwa H, Mwangi R, Mwerinde O, Olomi R, Drakeley C and Whitty CJ M.Rapid diagnostic tests compared with malaria microscopy for guiding outpatient treatment of febrile illness in Tanzania: randomised trial. BMJ 2007;334;403

55. She RC, Rawlins ML, Mohl R, Perkins SL, Hill HR, Litwin CM. Comparison of immunofluorescence antibody testing and two enzyme immunoassays in the serologic diagnosis of malaria. J Travel Med 2007; 14: 105-111

56. Chotivanich K, Silamut K, Day NPJ. Laboratory diagnosis of malaria infection-a short review of methods. Aust JMed Sci 2006; 27: 11-15.

57. Mharakurwa S, Simoloka C, Thuma PE, Shiff CJ, Sullivan DJ. PCR detection of Plasmodium falciparum in human urine and saliva samples. Malar J. 2006 Nov 8; 5:103.

58. Masika PM, Semarundu WJ, Urassa R, Mosha J, et al. Over-diagnosis of malaria is not a lost cause. Malaria Journal 2006, 5:120 doi:10.1186/1475-2875-5-120 